\title{
INVESTIGACIONES
}

\section{Modelo pedagógico de formación holística ambiental en los estudiantes de carreras pedagógicas del área de Ciencias Naturales en Cuba}

\author{
Pedagogical model of holistic environmental formation \\ in the students of pedagogical careers in the area of Natural Sciences in Cuba
Yunior Ramón Velázquez Labrada ${ }^{a}$, Eumelia Victoria Romero Pacheco ${ }^{b}$, Oscar Sigas Costafreda ${ }^{c}$, Mayelin Pérez Benitez ${ }^{d}$ \\ ${ }^{a}$ Centro de Estudios Multidisciplinarios de Zonas Costeras (CEMZOC), Universidad de Oriente, \\ Santiago de Cuba. yunior.velazquez@uo.edu.cu \\ ${ }^{b}$ Facultad de Ciencias Naturales y Exactas, Universidad de Oriente, Santiago de Cuba. \\ eumelia@uo.edu.cu \\ ${ }^{c}$ Facultad de Ciencias Sociales, Universidad de Oriente, Santiago de Cuba. \\ oscar.sigas@uo.edu.cu \\ ${ }^{d}$ Centro Universitario Municipal San Luis. Universidad de Oriente, Santiago de Cuba. \\ mayelin.perez@uo.edu.cu
}

\begin{abstract}
RESUMEN
El artículo aporta un modelo pedagógico de formación holística ambiental en los estudiantes de carreras pedagógicas del área de Ciencias Naturales en Cuba. En correspondencia con la metodología cualitativa, se aplicaron métodos como el holístico- dialéctico, análisis- síntesis, inductivo- deductivo durante la fundamentación epistemológica del objeto de estudio, así como la modelación en la construcción del modelo pedagógico. Se plantearon 6 configuraciones y 3 cualidades, integradas en las dimensiones: cognoscitiva contextual, educativa contextualizada y comportamental de la formación holística ambiental. Los resultados obtenidos permiten concluir que al confrontar la caracterización socio- ambiental contextual con la asimilación de saberes ambientales, se propicia la complementación interactiva de saberes ambientales, de la cual emerge la reflexión holística ambiental contextual y la capacidad proactiva holística ambiental, que se sintetizan en el desempeño pedagógico holístico ambiental. Constituyen cualidades resultantes: la proyección cognoscitiva ambiental integradora, la actitud reflexiva holística ambiental y el comportamiento cosmovisivo holístico ambiental.
\end{abstract}

Palabras claves: Holismo ambiental, conocimiento ambiental, actitud ambiental, educación ambiental.

\begin{abstract}
The article provides a pedagogical model of holistic environmental formation in the students of pedagogical careers of the area of Natural Sciences in Cuba. In correspondence with the qualitative methodology, methods such as holistic- dialectic, analysis- synthesis, inductive- deductive were applied during the epistemological foundation of the object of study, as well as modeling in the construction of the pedagogical model. 6 configurations and 3 qualities were proposed, integrated in the dimensions: contextual cognitive, contextualized educational and behavior environmental holistic formation. The results obtained allow us to conclude that by confronting the contextual partner-environmental characterization with the assimilation of environmental knowledge, the interactive complementation of environmental knowledge is fostered, from which emerges the contextual environmental holistic reflection and the holistic environmental proactive capacity, which are synthesized in the holistic environmental pedagogic performance. They constitute resulting qualities: the integrative cognitive environmental projection, the holistic environmental reflexive attitude and the holistic environmental cosmovisory behavior.
\end{abstract}

Key words: Environmental holism, environmental knowledge, environmental attitude, environmental education. 


\section{INTRODUCCIÓN}

En la actualidad, la magnitud de los problemas ambientales, con énfasis en el cambio climático, pone en peligro la supervivencia del hombre, lo cual exige un pensamiento reflexivo, global e integrador ante los múltiples eventos que ocurren a su alrededor, para mitigar los impactos negativos de su propio accionar en los distintos contextos de actuación. En este sentido, la Agenda 2030 y los Objetivos de Desarrollo Sostenible, promulgados por la Organización de las Naciones Unidas (CEPAL, 2015), en el objetivo número 4 Educación de calidad, plantea la necesidad de asegurar que todos los alumnos adquieran los conocimientos teóricos y prácticos necesarios para promover el desarrollo sostenible, entre otras cosas mediante la educación para el desarrollo sostenible y los estilos de vida sostenibles. También el objetivo 13 Acción por el clima, proyecta mejorar la educación, la sensibilización y la capacidad humana e institucional respecto de la mitigación del cambio climático, la adaptación a él, la reducción de sus efectos y la alerta temprana.

Estudios científicos en Cuba, prevén el incremento de la frecuencia e intensidad de ocurrencia de los fenómenos meteorológicos, así como la sobreelevación del nivel medio del mar en unos $27 \mathrm{~cm}$ hasta el 2050, trayendo como consecuencia una disminución lenta de la superficie emergida del territorio nacional en unos $2691.47 \mathrm{~km} 2$ (2.4\%). Para el 2100 se espera que se pueda producir un aumento del nivel medio del mar de hasta $85 \mathrm{~cm}$ y una pérdida de $6371.05 \mathrm{~km} 2(5.8 \%)$ de la superficie del archipiélago, trayendo como consecuencia, la creciente salinización de los acuíferos por el avance de la cuña salina. (Ministerio de Ciencia Tecnología y Medio Ambiente. CITMA, 2016).

También se estima que el clima de la isla, a largo plazo (2050-2100), sea más árido y extremo, caracterizado por prolongadas sequías y severos déficits de agua. Los paisajes secos de la región oriental se intensificarán y avanzarán progresivamente hacia la zona occidental, produciéndose la transformación del clima tropical húmedo a un clima subhúmedo seco, con amenazas de procesos de desertificación. (Planos, 2014). Ante esta situación, fue aprobado en abril de 2017, el Plan de Estado para el enfrentamiento al cambio climático en Cuba, en cuya Tarea 10, intenciona priorizar las medidas y acciones para elevar la percepción del riesgo, aumentar el nivel de conocimiento y el grado de participación de toda la población en el enfrentamiento al cambio climático y una cultura que fomente el ahorro del agua. (CITMA, 2017).

En correspondencia con lo planteado, la Constitución de la República de Cuba (Ministerio de Justicia, 2019), en el artículo 16, inciso f, promueve la protección y conservación del medio ambiente y el enfrentamiento al cambio climático que amenaza la sobrevivencia de la especie humana, sobre la base del reconocimiento de responsabilidades comunes. En este sentido, es necesario considerar la formación ambiental de las diferentes generaciones de estudiantes universitarios que se preparan como profesores, como una de las vías esenciales para contribuir a la protección óptima del entorno y la utilización racional de los recursos naturales, pues como futuros profesionales deberán incidir de manera positiva en la educación integral de otras personas.

Para el logro de lo antes planteado, se parte de considerar las investigaciones precedentes y experiencias de autores extranjeros en relación a lo ambiental, como: Novo (1998), Leff (2000), Sotolongo (2002), Delgado (2005), Simões, Yanes \& Moreira, (2018), que constituyen referentes teóricos para el tratamiento a lo ambiental en las Ciencias Naturales. Además, se significan autores nacionales como: Torres (1996), en relación a la educación 
ambiental; Díaz (1998), en la didáctica para contribuir a la protección de la fauna silvestre; Santos (2002), referido a la formación continuada en educación ambiental para docentes; Roque (2003), desde la formación de la cultura ambiental en los profesionales cubanos de nivel superior; Mc Pherson (2004), con propuestas para la educación ambiental en la formación de profesores y Cervantes (2006), conducente a la formación ambiental del ingeniero electricista.

Son también importantes los resultados investigativos de Romero (2010), en torno a la estrategia curricular de educación ambiental en las carreras pedagógicas; Fernández (2012), orientado hacia la formación ambiental del estudiante de Psicología desde la extensión universitaria; Martínez (2013) basada en la formación ambiental del maestro primario orientada al desarrollo agrosostenible en condiciones de montaña; Rodríguez (2016), dirigida a la actitud pedagógica ambiental en estudiantes de Biología - Geografía. De igual manera Labrada (2017) y García (2017), con respecto al tratamiento de los aspectos de la localidad y a la gestión ambiental sociocomunitaria desde los Centros Universitarios Municipales.

A pesar de lo anterior, se requieren nuevas miradas acerca de la formación del estudiante, dirigidas hacia la integración de lo cognoscitivo, lo educativo y lo comportamental, para potenciar lo ambiental desde las posibilidades que ofrecen los procesos sustantivos en la universidad y con ello favorecer el desempeño pedagógico de los estudiantes. De ahí que en el presente trabajo se propone un modelo pedagógico de formación holística ambiental en los estudiantes de carreras pedagógicas del área de Ciencias Naturales. El mismo, constituye una contribución a la teoría pedagógica, pues posibilita superar la manera fragmentada en que son considerados los aspectos ambientales en la formación del estudiante y contextualiza en dicho campo el holismo ambientalista como integrante de la epistemología contemporánea.

En correspondencia con la metodología cualitativa de la investigación social, se aplicaron diversos métodos de investigación del nivel teórico como el holístico- dialéctico, análisis- síntesis, inductivo-deductivo, en el procesamiento de la información derivada del estudio bibliográfico relacionado con la formación ambiental, así como la modelación en la construcción del modelo pedagógico.

\section{FUNDAMENTACIÓN EPISTEMOLÓGICA DE LA FORMACIÓN HOLÍSTICA AMBIENTAL EN LOS ESTUDIANTES DE CARRERAS PEDAGÓGICAS DEL ÁREA DE CIENCIAS NATURALES}

La formación de los estudiantes de carreras pedagógicas es de gran importancia, a partir de las transformaciones que tienen lugar en la sociedad cubana y en la Enseñanza Media, para la cual se preparan. En el ámbito universitario, Fuentes (2004), se ha referido a la formación como una vía para la construcción de significados y sentidos que implica el desarrollo humano progresivo, una construcción individual y social, un sistema de procesos conscientes, de naturaleza holística y dialéctica. Se coincide con lo anterior, al reconocer no solo el logro de aspectos básicos en la personalidad del estudiante, sino el carácter holístico de dicha formación. Además, Horruitiner (2009), circunscribe la formación al proceso sustantivo desarrollado en las universidades con el objetivo de preparar de forma integral a los estudiantes según las características de cada carrera. De esta manera se connotan los procesos académico, laboral, investigativo y extensionista. 
Estudios Pedagógicos XLVII Nº 1: 371-390, 2021

MODELO PEDAGÓGICO DE FORMACIÓN HOLÍSTICA AMBIENTAL EN LOS ESTUDIANTES DE CARRERAS PEDAGÓGICAS DEL ÁREA DE CIENCIAS NATURALES EN CUBA

En este sentido, la categoría formación, en el perfil pedagógico está encaminada hacia la construcción del rol profesional, posibilita el desarrollo de habilidades propias del ejercicio profesional en los diferentes niveles del sistema educativo (Martínez, 2013). La misma tiene lugar en la universidad y en las unidades docentes, donde los estudiantes realizan las prácticas en cada año académico, cuyo resultado es la preparación integral para el ejercicio de la profesión en los distintos contextos de actuación escolar. En este período se gesta el modo de actuación profesional, por ende, el proceso formativo debe estar en función de lograr la integralidad del futuro profesor, considerar las necesidades educativas, la integración de saberes básicos como saber ser, saber hacer y saber convivir.

El proceso de formación integral expresa la pretensión de centrar el quehacer de las universidades en la formación de valores en los profesionales de forma más plena, dotándolos de cualidades de alto significado humano, capaces de comprender la necesidad de poner sus conocimientos al servicio de la sociedad en lugar de utilizarlos solo para su beneficio personal. Implica también la necesidad de lograr un profesional creativo, independiente, preparado para asumir su autoeducación durante toda la vida; que sea capaz de mantenerse actualizado (Horruitiner, 2009).

La definición mencionada, pondera lo axiológico en el estudiante, que visto desde la perspectiva humanista, reconoce la necesidad de una actitud para hacer, sin embargo pudiera considerarse el tránsito desde el plano teórico, hacia la disposición de un comportamiento transformador en los estudiantes, consecuente con la formación recibida, que sea muestra del equilibrio entre lo interno y lo externo, al tener en cuenta que el estudiante puede estar apto para realizar determinadas actividades y en la práctica no efectuarlas.

Los aspectos anteriores se consideran importantes, más cuando el estudiante debe desplegar un accionar positivo dirigido a la protección del medio ambiente, como espacio fundamental donde se concreta su desempeño en interacción con otros factores ambientales, en un tiempo irrepetible, lo cual requiere considerarlo en un sentido totalizador que favorezca la transformación individual, pedagógica y social en general.

Por ende, se considera como elemento básico en la formación del estudiante, el aspecto ambiental, que según Parada (2008) expresa el resultado de los procesos concretos inherentes a la relación sociedad-naturaleza, dadas de manera histórica en correspondencia con el nivel de desarrollo de la actividad práctico-social que la direcciona, en un nivel de relaciones materiales mucho más complejas que aquellas formas que le preceden y contentivo de las nuevas formas históricas de la relación medio ambiente-desarrollo. Esto denota su carácter general y se constituye en un sustento para el tratamiento de lo ambiental desde la dimensión pedagógica, aunque sería más efectivo si se connotara la influencia biopsíquica y sociocultural.

En consecuencia, es importante referirse a la formación ambiental en los futuros profesionales de la educación, la cual ha sido planteada como conjunto de actividades teóricas o prácticas, que denotan su carácter de proceso, orientada hacia la calidad (Figueroa, 1995). A dicho proceso, Roque (2003) añade su carácter integrador, además de incluir, como parte de esta, el sistema de valores, de conocimientos, la capacidad de planificar y de ejecutar la actividad profesional. En concordancia, Mc Pherson (2004), resignifica como permanente el carácter de dicha formación, desde los procesos sustantivos. Además, pondera lo reflexivo, lo creativo, lo ético, lo colectivo, desde la autogestión y la diversidad cultural, en tanto Martínez (2013), centra su atención en lo comunitario como parte de dicha formación. 
Desde esta óptica, para ser consecuente con la utilidad del conocimiento histórico en la preservación del medio, sería prudente atender las relaciones del hombre consigo mismo y con los demás, a partir una perspectiva socio-ambiental, en tanto se considera que para la comprensión de los problemas ambientales, se exige que la relación teoría - práctica, redunde en la calidad de vida del hombre.

El autor de esta investigación considera que la formación ambiental es un proceso integrador de conocimientos, hábitos, habilidades, capacidades, valores y valoraciones, que permite un pensamiento reflexivo en cada persona, según las características de la naturaleza humana condicionada por la época histórica, a la que debe responder mediante la socialización de sus experiencias, las que varían en el curso de la historia, se desarrolla, transforma y contextualiza en el proceso educativo en pro de un modo de actuación ambiental responsable, orientado hacia el desarrollo sostenible.

Se coincide con Roque (2003) que el desarrollo sostenible constituye un proceso de creación de las condiciones materiales, culturales y espirituales que propicien la elevación de la calidad de vida de la sociedad, con un carácter de equidad y justicia social de forma sostenida, basado en una relación armónica entre los procesos tanto naturales como sociales, cuyo objeto involucra a las actuales y futuras generaciones. No obstante, en esta definición tampoco queda debidamente explicitado lo referente a las condiciones biopsíquicas y sociales que inciden en la calidad de vida del hombre.

La calidad de vida como parte de lo expresado con anterioridad no es solo un componente del desarrollo sostenible, sino una condición indispensable para que este pueda ser alcanzado una vez que los ciudadanos cuenten con las condiciones básicas necesarias que se implican en el concepto de calidad de vida, cuyos componentes deben ser garantizados por el Estado, pues no se alcanza el futuro sin garantizar el presente, de modo que la proyección hacia el desarrollo sostenible pueda tener una base crítica, analítica, participativa, valorativa en relación con el entorno, como expresión de una posición holística que se concrete en la relación de la escuela con la vida.

En este sentido, se está de acuerdo con Velasco (2009), al referir que la holística implica una visión de los objetos, fenómenos, tanto naturales como sociales, en su totalidad, en su conjunto, en su complejidad, en interacción, desde una actitud integradora, que permita entender las particularidades y generalidades de la relación entre los procesos, los protagonistas y sus contextos, que por lo regular no se comprenden si se estudian por separado los aspectos que conforman el todo. Cada acontecer está relacionado con otros acontecimientos, los cuales producen entre sí nuevas relaciones en un proceso que compromete el todo, es decir lo que fue, lo que está siendo, lo que será, lo que está en posibilidad de ser.

Además, dicha autora considera que el pensamiento holístico transcurre desde lo general hacia lo particular y viceversa, se orienta hacia una cosmovisión con base en preceptos comunes para todos los seres vivos; implica una actitud abierta hacia la historia, la indagación, la comprensión, la apreciación de interpretaciones y posibilidades, percibir los contextos, las ideas y las situaciones en sus múltiples relaciones. No obstante, aunque dichos planteamientos se refieren a aspectos que son condiciones indispensables para el desarrollo de un pensamiento holístico, estos poseen un carácter muy general, sin esclarecer las premisas para que el mismo pueda contextualizarse en la educación de los diferentes grupos etáreos.

Por otro lado, Espino de Lara (2013), en relación a la concatenación entre los elementos que conforman la totalidad del universo, como aspecto significativo del holismo, que se 
opone a posiciones separatistas, reduccionistas, desequilibradas para dar apertura a un conocimiento que transita hacia la integridad de la conciencia; considera que lo planteado debe ser exponente de los diversos contextos en que se desarrollan los estudiantes, de lo significativo y que se manifieste en la comunicación, en la actuación y la subjetividad desde una visión transdisciplinaria para la comprensión de los procesos de la realidad.

Lo anterior connota la importancia de explicitar el carácter holístico que debe tipificar a la formación, para el logro de lo ambiental, que también tiene una esencia integradora y requiere intencionar una concepción dirigida a desarrollar un pensamiento totalizador acerca de la protección ambiental en los entornos, naturales y sociales, entendidos como el hábitat inmediato de interacción con los factores ambientales, a los cuales el hombre debe adaptarse y proteger, porque es parte de ese medio ambiente en su totalidad. Así, para que la formación sea holística, se debe propiciar la adquisición de aprendizajes específicos por parte de los estudiantes. Debe conducirlos más allá de los niveles alcanzados en un momento determinado de su vida, propiciar la realización de aprendizajes que superen las metas ya logradas y la creatividad en el tratamiento a las diferentes problemáticas ambientales.

Una mirada a la formación holística exige tomar en consideración lo planteado por Gallegos (2009), en cuanto a la educación holista, en tanto la misma debe incluir relaciones humanas significativas, basadas en el respeto, la humildad, la búsqueda de las mejores soluciones a los problemas, el bienestar individual, la armonía, el diálogo, la creación mutua de significados, la comprensión de los aspectos de la vida en su contexto, una visión integral del mundo, del propio hombre y del futuro. Incluye el respeto a la diversidad cultural, el reconocimiento de que el desarrollo humano requiere un conocimiento multidimensional, multinivel, ligado a una dimensión ético-espiritual, con alta sensibilidad humana y amor universal.

Además, sería oportuno fomentar el vínculo entre los conocimientos de las distintas disciplinas con el contexto socio histórico (Busquets, T., Silva, M. \& Larrosa, P., 2016). Así como, tener en cuenta la relación espacio-tiempo, como elemento fundamental en la asunción de una posición holística vinculada a la formación ambiental. Así el estudiante, a partir de su cosmovisión, incorpora de manera sistemática en su desempeño, la comparación de los diferentes momentos históricos de desarrollo de los procesos naturales y sociales y el tiempo promedio de impacto de la acción humana de manera negativa sobre estos espacios.

En este sentido, el autor define la formación holística ambiental como el proceso integrador de las influencias educativas en el estudiante, mediante el cual evoluciona hacia una comprensión cada vez más compleja del contexto socio-ambiental, que se evidencia en una proyección cognoscitiva sintetizadora de saberes acerca de los distintos objetos, fenómenos naturales y sociales con los que se relaciona de manera directa o indirecta, así como en una actitud reflexiva, que se concreta en un comportamiento cosmovisivo consecuente en relación con sus características individuales y los deberes ambientales a favor de su entorno.

En armonía con lo anterior, se asume que los modelos constituyen representaciones simplificadas de la realidad, donde se representan sus rasgos más esenciales, de acuerdo con el propósito del estudio realizado, ello es asumido como parte de los presupuestos teóricos para la elaboración del mismo, lo cual contribuye a develar nuevas relaciones y regularidades (Tamayo, 2015).

Además, se es consecuente con lo postulado por Vigotsky (1987) en relación a la mediación y la internalización, como parte de su Teoría Histórico Cultural, en tanto permite 
pensar que la formación ambiental del estudiante está mediada por aspectos cognoscitivos y educativos. De igual forma se tienen en cuenta los presupuestos teóricos en torno a la Pedagogía de la Educación Superior, desde la Concepción Científica de lo Holístico Configuracional de Fuentes (2011), como el basamento teórico - metodológico y método científico general en la construcción teórica que se presenta.

\section{DIMENSIONES Y CONFIGURACIONES DEL PEDAGÓGICO DE FORMACIÓN HOLÍSTICA AMBIENTAL EN LOS ESTUDIANTES DE CARRERAS PEDAGÓGICAS DEL ÁREA DE CIENCIAS NATURALES}

El modelo pedagógico de formación holística ambiental en los estudiantes de carreras pedagógicas del área de Ciencias Naturales, como representación teórica es considerado desde la integración de lo ontológico, lo epistemológico y lo lógico, con gran significado en el redescubrimiento del objeto que se estudia, por lo cual indaga en las relaciones dialécticas para determinar tanto las cualidades como las regularidades en el orden pedagógico que se presentan entre sus elementos constituyentes. Así, la naturaleza pedagógica en el modelo que se propone se evidencia en tanto se ajusta a las aspiraciones de la educación cubana, en su vínculo con el contexto social; en sus dimensiones imbrica lo instructivo, lo educativo y lo comportamental desde la perspectiva holística, insertado en el proceso de formación de estudiantes de carreras pedagógicas del área de Ciencias Naturales.

Dicho modelo pedagógico, consiste en la interpretación sintetizada de las particularidades esenciales del objeto y el campo estudiados, que emergen además de la sistematización cognoscente, permiten su generalización y sustenta la construcción teórica que configura y explica la formación holística ambiental en los estudiantes de carreras pedagógicas del área de Ciencias Naturales, expresadas a través de relaciones esenciales, las cuales permiten el ascenso a un nuevo nivel interpretativo de los referentes teóricos del objeto investigado. Estas relaciones caracterizan la modelación de la formación holística ambiental en los estudiantes de carreras pedagógicas del área de Ciencias Naturales, las cuales se revelan a través de tres dimensiones:

- Dimensión cognoscitiva contextual de la formación holística ambiental.

- Dimensión educativa contextualizada de la formación holística ambiental.

- Dimensión comportamental de la formación holística ambiental.

Dimensión cognoscitiva contextual de la formación holística ambiental: Esta dimensión es expresión de la relación dialéctica entre las configuraciones en pares dialécticos: caracterización socio-ambiental contextual y la asimilación de saberes ambientales, que está mediada en su síntesis por la complementación interactiva de saberes ambientales y se obtiene como cualidad resultante la proyección cognoscitiva ambiental integradora.

La caracterización socio-ambiental contextual, como configuración, plantea si el estudiante es capaz de reconocer los conocimientos que posee acerca del medio ambiente, los problemas ambientales y su dinámica: definición, causas, consecuencias, medidas de solución; percibir transformaciones en la relación sociedad-naturaleza, para transitar de conclusiones iniciales no definitivas hacia otras más acabadas. También si profundiza y socializa de manera adecuada la información ambiental e interactuar de manera correcta en 
grupos de iguales. Esta configuración, es expresión de la confluencia del estudiante consigo mismo, con su grupo de iguales, con su entorno natural, social y con lo histórico-cultural enmarcado en un medio ambiente específico, según su propia cosmovisión, por lo que está dada por la concreción en un espacio y tiempo determinados.

De ahí que lo socio-ambiental, desde lo pedagógico, garantiza poder transmitir conocimientos para una asimilación razonada del ambiente, en el proceso formativo, que implica el reconocimiento de los recursos naturales como sustento de las culturas autóctonas y como condición de sobrevivencia, garante del futuro especialista en el área de Ciencias Naturales. Lo contextual identifica los escenarios donde los estudiantes interactúan, donde se establecen un conjunto de relaciones sociales. En este sentido, la caracterización socioambiental contextual de los estudiantes será realizada por el docente y al mismo tiempo por el estudiante, la que puede expresarse en relación a sí mismo y a su contexto de origen, a partir de las cuales se revelan las potencialidades o carencias presentes en ellos. Esta caracterización se actualizará en cada año académico.

En la identificación de la formación holística ambiental, la caracterización socioambiental contextual se dinamiza por la integración que se establece entre la realidad cognoscitiva ambiental del estudiante, la utilización de la información ambiental disponible y la interacción en grupos de iguales, como rasgos de esta configuración.

La realidad cognoscitiva ambiental del estudiante está determinada por los conocimientos teóricos que posee el mismo acerca del medio ambiente en general y del entorno en particular, en un momento concreto. Tiene en cuenta lo fáctico, es decir, los datos asociados a objetos, procesos, fenómenos naturales y sociales, noticias, hechos significativos, relacionados con el medio ambiente en general o el entorno en particular, cuya apropiación se apoya en una lógica reproductiva, así como aquellos conocimientos que posee, a partir de conceptos y explicaciones lógico-conceptuales, incorporados a través de la escuela, la familia, los entes comunitarios, entre otras fuentes.

También, incluye la incorporación del saber ambiental, contentivo de conocimientos, hábitos, habilidades, capacidades, valores y valoraciones relacionadas con el medio ambiente, que son incorporados de manera sistémica, novedosa y lógica, a través del contenido de las clases en las diversas asignaturas, según las potencialidades de las mismas, las exigencias del modelo del profesional, así como las estrategias educativas por año académico. Los contenidos relacionados con el medio ambiente como parte del componente académico tienen una contextualización práctica en lo laboral, investigativo y extensionista, lo cual se debe intencionar desde el colectivo pedagógico. Contempla el análisis de lo empírico y lo normativo ambiental necesarios para ser procesados, en un proceso autoreflexivo, de intersubjetividad que implica aprender a aprender con otros, en el diálogo de saberes, en un contexto de interculturalidad donde se define la particularidad de cada actuación ambiental.

La realidad cognoscitiva ambiental tiene una expresión en la utilización de la información ambiental disponible, que deviene en un elemento importante en la caracterización socioambiental contextual.

La utilización de la información ambiental disponible incorpora la manera en que los estudiantes emplean y desarrollan la información ambiental en la actividad intelectual, valorativa y práctica. Además, cómo aplica los conocimientos adquiridos, básicos para la adopción de determinadas posturas ambientales según el contexto, si socializa la información en el grupo escolar. 
Lo anterior se complementa con la interacción en grupos de iguales, que refiere las relaciones establecidas entre los estudiantes de un mismo grupo escolar y pueden ser determinantes para el logro de prácticas ambientales adecuadas. En este contexto, donde todos poseen la condición de estudiantes, el mismo rango de edad y una marcada aspiración profesional, se manifiestan diversas relaciones sociales.

En este sentido, la interacción debe ocurrir en un clima no egocéntrico, sino abierto al reconocimiento de cada uno como parte activa del medio, a la construcción colectiva de saberes, la incorporación de las mejores experiencias derivadas de la historia de vida individual, así como la demostración de la autorregulación alcanzada en la continuidad de la convivencia a este nivel, la disposición para la cooperación, lo que deviene en un equilibrio consigo mismo, así como en relación a sus semejantes. Eso facilitaría la adopción de medidas personales y colectivas, a favor del entorno.

De este modo, la caracterización socio-ambiental contextual, adquiere un sentido integrador de los aspectos teórico-prácticos en relación con el medio ambiente, que propicia la caracterización socio-ambiental contextual del estudiante, sin embargo, desde esta configuración no es posible acceder a la totalidad de los elementos requeridos para la formación holística ambiental, por lo cual se requiere transitar hacia la configuración asimilación de saberes ambientales.

La configuración asimilación de saberes ambientales es entendida como el modo a través del cual los estudiantes incorporan nuevos saberes a sus representaciones mentales preexistentes. Permite el tránsito desde una reproducción del conocimiento ambiental, que tiene lugar a partir de las representaciones mentales según la influencia externa anterior, sin requerir de la presencia del objeto o fenómeno de la realidad, hacia una aplicación de dicho saber ambiental, donde, desde un mismo conocimiento, encuentra diferentes alternativas para la mitigación o solución de problemas ambientales presentes en su entorno inmediato, como expresión de su cosmovisión.

Así se arriba a la reconstrucción del conocimiento ambiental, resultado de la concepción de los significados, conceptos e ideas con un sentido abstracto y generalizador; revelador de la esencia de los objetos, fenómenos y nexos entre ellos. Dicha asimilación, en el estudiante, ocurre según las experiencias histórico-culturales de su contexto, de su propia vida, a partir de las cuales recepciona y decodifica la información en dependencia del significado atribuido. En correspondencia, lo construido se percibe primero por los órganos de los sentidos y se asimila, si él está preparado para ello.

En la dimensión cognoscitiva contextual de formación holística ambiental, la asimilación de saberes ambientales se dinamiza a través de la integración entre la recepción de saberes ambientales, la apropiación de saberes ambientales y la reestructuración valorativa de saberes ambientales.

La recepción de saberes ambientales consiste en aquellos conocimientos, que el estudiante recibe por diferentes vías, los cuales relaciona con sus vivencias, hábitos, habilidades, valores y valoraciones, contextualizados al entorno y en función de lo cual le atribuye una determinada importancia para su proceso formativo. Se expresa, además, en la manera en que el estudiante capta el medio ambiente en su realidad factual, procesa las informaciones disponibles, establece las variadas conexiones entre las exigencias sociales y las acciones que se generan en la práctica.

La recepción de saberes ambientales asegura la apropiación de saberes ambientales, la que se erige como el proceso y resultado de la aprehensión por el estudiante de los 
Estudios Pedagógicos XLVII Nº 1: 371-390, 2021

MODELO PEDAGÓGICO DE FORMACIÓN HOLÍSTICA AMBIENTAL EN LOS ESTUDIANTES DE CARRERAS PEDAGÓGICAS DEL ÁREA DE CIENCIAS NATURALES EN CUBA

saberes asociados al medio ambiente. La apropiación de saberes ambientales se manifiesta en la medida que el estudiante demuestra un saber sistematizado, relaciona los nuevos conocimientos con los previos, como evidencia de la generalización de ideas, es decir, todo lo que realice debe estar en correspondencia con lo aprendido, lo cual forma parte de los estereotipos dinámicos de la persona, demuestra una disposición positiva hacia el medio ambiente, según la herencia cultural acumulada. Una vez que en el estudiante ha tenido lugar la recepción y apropiación de saberes ambientales, están dadas las condiciones de tránsito hacia la reestructuración valorativa de saberes ambientales, lo que proporcionará el reordenamiento de los conocimientos incorporados, según la diversidad de contextos en el cual se desarrolla.

La misma se expresa en el reordenamiento del sistema de ideas preestablecidas, que deviene en la atribución de nuevos significados, contextualizados a las características de los contextos en los cuales participa y se constituyen en nuevas formas de percibir la realidad. Permite la corrección de aquellos elementos cognitivos asumidos de forma incorrecta, para trascender hacia nuevas formas de concebir la realidad, sobre la base de las experiencias y vivencias asociadas a esos nuevos conocimientos.

Para la formación holística ambiental en los estudiantes de carreras pedagógicas del área de Ciencias Naturales, es necesario considerar la complementación interactiva de saberes ambientales como síntesis de la relación dialéctica entre la caracterización socio- ambiental contextual y la asimilación de saberes ambientales. En este caso, el estudiante debe ser capaz de redimensionar el conocimiento ambiental actual, según la diversidad de contextos en los cuales participa y la interacción con las personas; como expresión de la significación e interacción de saberes ambientales. Es decir, debe manifestar una comprensión cada vez más compleja del contexto en que se desarrolla, lo cual demuestra a través del diálogo sistemático, entre otras formas. En la configuración complementación interactiva de saberes ambientales se concreta la relación de integración entre los rasgos comprensión de saberes ambientales, significación de saberes ambientales e independencia cognoscitiva integrativa ambiental.

La comprensión de saberes ambientales es el proceso mediante el cual el estudiante entiende los contenidos acerca del medio ambiente y el alcance de los problemas ambientales, el reconocimiento de las relaciones entre ellos, con énfasis en sus causas objetivas, la interpretación de su significación contextual en su totalidad, no de manera aislada. Ello propicia la formulación de supuestos (anticipar temas, propósitos, inferir información del contexto), que les permitan concebir como positiva o negativa la realidad ambiental existente. Considerar algo como positivo o normal, implica reconocer sus beneficios para su incorporación consciente como saberes, entender los procesos básicos que determinan modos de convivencia y los fenómenos asociados al objeto que se analiza.

La significación de saberes ambientales en los estudiantes consiste en un proceso sistemático para atribuirle a los hábitos, habilidades y valores nuevos sentidos a partir del conocimiento ambiental, que le ofrezca las posibilidades para la interacción entre los conocimientos, necesidades, intereses, motivaciones y experiencias individuales con un nuevo significado. De esta manera, cuando el saber ambiental provoca satisfacción en el estudiante, favorece la adquisición de nuevos conocimientos y la ampliación de su cosmovisión, para actuar en su entorno inmediato. Ello genera motivaciones para profundizar en estos contenidos, alcanzar mejor estatus personal intelectual y estar en condiciones para la orientación positiva a favor de la solución o mitigación de determinadas problemáticas individuales o grupales. 
La independencia cognoscitiva integrativa ambiental, se manifiesta en la armonía entre los conocimientos, hábitos, habilidades, valores y valoraciones en el estudiante, que le permiten establecer el vínculo de lo aprendido con las prácticas ambientales más frecuentes en los distintos contextos en que habita. Se evidencia en el razonamiento acertado acerca de las medidas para mitigar o adaptarse al impacto de problemas ambientales y en la expresión en el discurso de criterios a favor del cuidado del entorno.

Del sistema de relaciones que se establece entre las diferentes configuraciones que expresan la naturaleza de la dimensión antes mencionada, emerge como cualidad resultante la proyección cognoscitiva ambiental integradora, la cual es expresión esencial de la preparación cognoscitiva ambiental que posee el estudiante para aplicarla en la práctica.

Esta considera al estudiante con suficientes potencialidades para orientar su actividad en relación a sí mismo, con su grupo de iguales y su contexto socio-profesional, a partir de los conocimientos, hábitos, habilidades, valores, valoraciones y capacidades con los cuales está en condiciones de operar, hacia la identificación de los problemas ambientales del entorno, en pro de plantear acciones transformadoras desde la perspectiva de sostenibilidad.

La relación dialéctica entre la caracterización socio-ambiental contextual, la asimilación de saberes ambientales y la complementación interactiva de saberes ambientales, propicia el ascenso hacia la dimensión cognoscitiva contextual de la formación holística ambiental, que se revela para propiciar un momento cualitativo superior de interpretación de la misma.

La dimensión cognoscitiva contextual de la formación holística ambiental (Figura 1) es una configuración que expresa la cualidad que adquiere esta, al proyectarse hacia el logro de una formación holística ambiental. Así lo cognoscitivo contextual tiene una expresión en la caracterización socio-ambiental contextual, pues si bien el estudiante no es el contexto en sí, es el resultado de las diferentes condicionantes de ese contexto, donde adquiere y desarrolla los conocimientos, hábitos, habilidades, valores, valoraciones y capacidades que emplea en su radio de acción.

Ello ocurre en la medida que el estudiante conoce sus propias carencias cognoscitivas ambientales, las cuales soluciona a partir de asimilar y complementar los saberes ofrecidos, en función de proyectarse hacia un nuevo estado de exploración en los saberes ambientales integrados, que dinamicen su práctica cotidiana. La representación gráfica del sistema de relaciones entre las configuraciones de la dimensión abordada se representa en la figura 1.

Figura 1. Dimensión cognoscitiva contextual de la formación holística ambiental en los estudiantes de carreras pedagógicas del área de Ciencias Naturales.

Caracterización socio- ambiental contextual Asimilación de saberes ambientales
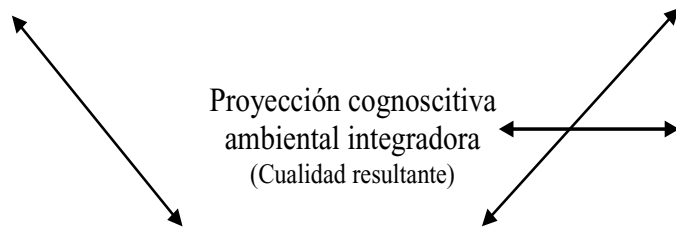

Dimensión cognoscitiva contextual de la formación holística

Complementación interactiva de saberes ambientales ambiental

Fuente. Elaboración propia. 
Estudios Pedagógicos XLVII Nº 1: 371-390, 2021

MODELO PEDAGÓGICO DE FORMACIÓN HOLÍSTICA AMBIENTAL EN LOS ESTUDIANTES DE CARRERAS PEDAGÓGICAS DEL ÁREA DE CIENCIAS NATURALES EN CUBA

La complementación interactiva de saberes ambientales en calidad de síntesis de la relación dialéctica entre la configuración caracterización socio- ambiental contextual y la asimilación de saberes ambientales, constituye a la vez, expresión de un nuevo movimiento dentro de la formación holística ambiental, en tanto deviene en síntesis de dos nuevas configuraciones: la reflexión holística ambiental contextual y la capacidad proactiva holística ambiental.

En la reflexión holística ambiental contextual el estudiante debe ser capaz de razonar, analizar de manera lógica, identificar, comparar y clasificar conceptos; construir criterios valorativos de las representaciones ambientales construidas de forma mental, establecer relaciones causales, conclusivas e inferencias a partir de las mismas. Es decir, debe pensar de manera activa y totalizadora sobre lo ya pensado en relación a las problemáticas ambientales del entorno y deducir lo correcto e incorrecto en el accionar cotidiano. Lo demuestra en su disposición y adopción de actitudes preventivas a favor del entorno.

La configuración reflexión holística ambiental contextual deviene entonces, desde lo cognoscitivo contextual, como sustento en este proceso y se dinamiza a través de los rasgos percepción ambiental contextualizada, disposición armónico ambiental y prevención ambiental contextualizada. Es entendida como un proceso psíquico a través del cual, en el estudiante se armonizan los componentes endógenos y exógenos, mediante sus sentidos, para reflejar los rasgos más significativos de la personalidad como un todo, en función de los que desarrolla su percepción valorativa acerca del entorno. A partir de este proceso se forma la imagen integral, acerca de los componentes bióticos, abióticos y socioeconómicos del medio ambiente, con sus características y las relaciones que los unen. Permite recibir, elaborar e interpretar en cada momento concreto, la información procedente de sí mismo y su entorno.

La disposición armónico ambiental se expresa en la decisión del estudiante de ser receptivo, al comprender sus carencias, estar dispuesto al cambio positivo en sí mismo y en el entorno, así como a un actuar consciente a favor del medio ambiente como respuesta a la asunción de los saberes ambientales. La disposición que el estudiante adopta ante la realidad contempla los objetos concretos, situaciones, ideas, fenómenos sociales y está en dependencia de las características de la personalidad de los mismos, de su percepción acerca de lo que es favorable o desfavorable, lo cual puede apreciarse en el estado emocional, que demuestra durante la valoración de los objetos y/o situaciones, según los saberes ambientales adquiridos en relación con los mismos.

La prevención ambiental contextualizada se concibe como el proceso sistemático y activo que actúa como un mecanismo regulador en el accionar del estudiante, en función de evitar la autoprovocación de problemas ambientales, anticiparse a la ocurrencia de hechos que afecten el entorno, de modo que pueda emitir criterios de alerta a tiempo, o disminuir las consecuencias, contrarrestar la proliferación de dichos problemas ambientales en su radio de acción y en todo caso estar en condiciones de adoptar alternativas de solución o mitigación apropiadas ante una situación ambiental dada.

La reflexión holística ambiental contextual se articula con la capacidad proactiva holística ambiental, que como configuración se revela en la dinámica (rapidez, facilidad, profundidad, precisión, originalidad, constancia y calidad) que posee cada estudiante, para de forma individual, adquirir, asimilar y aplicar en su actividad los conocimientos, hábitos, habilidades y valores relacionados con el medio ambiente, como expresión del dominio de las operaciones mentales que están en la base de los mismos. En tal sentido, esta configuración 
se dinamiza a través de los rasgos reconocimiento de la aptitud ambiental, combinación de capacidades proambientales y regulación cosmovisiva contextual ambiental.

El reconocimiento de la aptitud ambiental consiste en que el estudiante, a partir de sus particularidades biológicas, reconozca sus propias limitaciones y/o potencialidades y en función de ello se autoconsidere apto en lo físico e intelectual, para llevar a la práctica aquellas acciones preventivas que dispuso realizar a favor de su entorno. Se manifiesta además en la medida que es capaz de cumplir las exigencias que le impone una determinada actividad o es capaz de indagar acerca de las ayudas necesarias para realizarlas con éxito.

Este rasgo se complementa con la combinación de capacidades proambientales, que se evidencia en la manera en que cada estudiante combina las cualidades de sus propias capacidades con las de otros miembros de su grupo de iguales, para realizar con éxito diferentes actividades a favor del medio ambiente. En relación con las capacidades se encuentran las cualidades principales, básicas para la realización de una actividad específica relacionada con el medio ambiente, tales como la observación de objetos y fenómenos naturales, la búsqueda de explicaciones y soluciones a un determinado problema ambiental. Existen cualidades auxiliares que, aunque no estén presentes, sino compensadas con otras, contribuyen al éxito de la actividad, por ejemplo, la planificación de acciones, la organización, el control y la evaluación de las medidas proambientales, en un contexto dado.

La regulación cosmovisiva contextual ambiental se manifiesta como un catalizador de todo cuanto ha podido asimilar el estudiante según lo recibido con antelación y se expresa en una posición cosmovisiva más compleja para articular sus conocimientos. Es su capacidad para intencionar de forma consciente su actuación ambiental, según los objetivos, los motivos y las vías más apropiadas para ello, en lo cual interviene lo cognoscitivo y lo afectivo. Ello está en correspondencia con la manera en que refuerza sus hábitos, habilidades, valores y valoraciones para el logro de lo ambiental.

En este sentido, los hábitos para el logro de lo ambiental consisten en la automatización en la ejecución y regulación de las operaciones dirigidas a la protección y cuidado del entorno. Es decir, la automatización de procedimientos para la realización de las diversas acciones, mientras que las operaciones son las formas de realización de las acciones según las condiciones del contexto.

Las habilidades para el logro de lo ambiental son expresión del dominio y el resultado sistematizado de las acciones psíquicas y prácticas que posee el estudiante, que le permiten una regulación racional de la actividad a favor del cuidado, protección del entorno y mitigación de los problemas ambientales.

La realización de las acciones que como estructura interna comprende la habilidad, requiere de la regulación consciente por el estudiante, para llevar a la práctica los conocimientos y los métodos adquiridos con el fin de proteger el medio ambiente.

Los valores proambientales están presentes en el estudiante cuando es capaz de manifestar en público su desacuerdo con las prácticas ambientales negativas, mantiene una reiterada reacción positiva ante el cuidado y protección del entorno, producto de la aprehensión por él de las condiciones del entorno natural, de la intersubjetividad que provoca aprender a aprender en la colectividad, mediante el diálogo de saberes en circunstancias de interculturalidad, que definen lo peculiar en cada situación ambiental.

De las relaciones dialécticas que se establecen entre las configuraciones reflexión holística ambiental contextual, capacidad proactiva holística ambiental y complementación interactiva de saberes ambientales, emerge como cualidad resultante la actitud reflexiva holística 
ambiental, la cual expresa la predisposición o disposición del estudiante para reaccionar de manera favorable ante determinados objetos, situaciones, componentes del medio ambiente, que incluye a las demás personas, a partir de la integración de saberes y motivos.

La relación dialéctica que se establece entre la complementación interactiva de saberes ambientales, la reflexión holística ambiental contextual y la capacidad proactiva holística ambiental, propician el ascenso hacia la dimensión educativa contextualizada de la formación holística ambiental, lo cual revela un salto cualitativo superior de interpretación del proceso que se modela.

Dicha dimensión (Figura 2) se expresa en la manera en que el estudiante razona, analiza e integra de manera lógica un pensamiento activo y reflexivo, que consecuente con los saberes ambientales incorporados resultan adecuados para una toma de decisiones; es una demostración totalizadora de los procedimientos inherentes al desarrollo de capacidades, así como una actitud reflejo de la disposición para una autorregulación contextualizada a la realidad educativa ambiental en que se desarrolla.

Figura 2. Dimensión educativa contextualizada de la formación holística ambiental en los estudiantes de carreras pedagógicas del área de Ciencias Naturales.

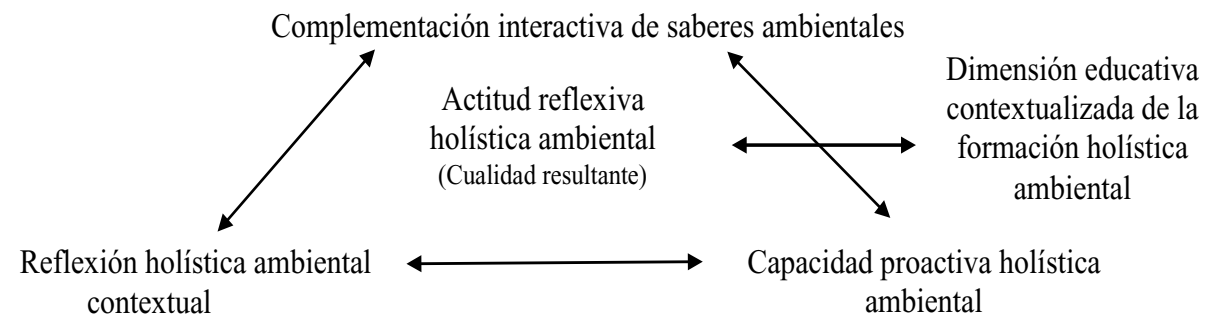

Fuente. Elaboración propia.

Por otro lado, puede entenderse que para la formación holística ambiental del estudiante de carreras pedagógicas del área de Ciencias Naturales, no es suficiente con la complementación interactiva de saberes ambientales como resultado de la relación entre la reflexión holística ambiental contextual y la capacidad proactiva holística ambiental, por ende, es necesario que su accionar se concrete en un desempeño pedagógico holístico ambiental que como síntesis entre la reflexión holística ambiental contextual y la capacidad proactiva holística ambiental, da cuenta del comportamiento cosmovisivo holístico ambiental como cualidad resultante, en la nueva dimensión comportamental de la formación holística ambiental.

El desempeño pedagógico holístico ambiental, está dado por la relación teórico-práctico ambiental en el estudiante, que no solo le permita explicar los fenómenos de la realidad objetiva, de acuerdo a su cosmovisión y las reflexiones en torno a ella, sino demostrar un accionar positivo, encaminado hacia la exploración de la diversidad de alternativas para la transformación de sí mismo, de la colectividad, del entorno natural y social desde una perspectiva sostenible, dado que la actividad pedagógica con un enfoque holístico ambiental debe transcurrir desde la formación inicial hasta la formación permanente, lo cual garantiza la correcta actuación de los egresados en los diferentes contextos de actuación. 
En este sentido, el desempeño pedagógico holístico ambiental, es expresión de las cualidades y las actitudes concretas del estudiante, no como resultado de un proceso formativo tradicional, sino como un proceso activo, ordenado y sistemático de apropiación constructiva de saberes vinculados a la sociedad en general y a la profesión en particular, donde se demuestre de forma práctica el saber hacer, el saber ser y el saber convivir, mediante su actividad transformadora con un carácter sostenible y creativo. Como rasgos esenciales de esta configuración se muestran la adaptabilidad a la diversidad contextual ambiental, la convivencia ético profesional ambiental y la transferencia de experiencias ambientales en la diversidad contextual.

La adaptabilidad a la diversidad contextual ambiental constituye el proceso y resultado que promueve en el estudiante la capacidad para adaptarse a las características de los diversos entornos en que cohabita con su grupo de iguales en edades e intereses y al mismo tiempo diverso en su esencialidad, así como al impacto de los problemas ambientales, para en consecuencia aportar respuestas adecuadas y pertinentes a favor del medio.

Si bien las adaptaciones poseen un carácter variable, según las características de los factores abióticos, sociales, culturales y económicos de cada lugar, el estudiante al adaptarse debe separarse de determinadas prácticas ambientales, desaprobadas en el espacio geográfico donde desea coexistir, y adquirir nuevas prácticas en correspondencia con lo que se espera de él y con las habilidades sociales que posee. Es decir, debe adecuar sus modelos de desempeño a las exigencias de su medio social, en función de la transformación positiva del entorno.

La convivencia ético profesional ambiental se da en la correcta interacción entre los estudiantes y entre estos con los restantes factores ambientales, como concreción del saber ser, saber hacer y saber convivir en el respeto a límites establecidos. En correspondencia con lo planteado, se considera importante que el estudiante conozca y practique el respeto a los límites en la convivencia como el respeto a la individualidad de cada persona, a la construcción colectiva, a la toma de decisiones correctas, a estar dispuesto a escuchar los señalamientos y sugerencias. Así la interacción se entiende como todo nexo o actividad entre las personas y con el medio ambiente.

También el medio social establece límites espaciales donde los estudiantes más que hacer uso desmedido de lo físico existente, deben velar por la sostenibilidad, por la socialización basada en el respeto a la identidad, que conlleve a mejores relaciones de convivencia en relación con el entorno, más que a la imposición de normas y patrones improductivos. Así, como parte de la convivencia, se encuentran las interacciones personales, es decir, el contacto directo o indirecto, entre los estudiantes mediado por la comunicación. Esto contempla la influencia recíproca de la trasmisión y recepción de la información ambiental, así como la expresión de emociones, estados de ánimo, sentimientos, basados en el respeto a la individualidad, de modo que se potencien relaciones de afecto y compromiso.

En la formación holística ambiental en los estudiantes de carreras pedagógicas, desde el punto de vista de las relaciones que se establecen entre las configuraciones reflexión holística ambiental contextual, la capacidad proactiva holística ambiental y el desempeño pedagógico holístico ambiental, emerge como cualidad resultante el comportamiento cosmovisivo holístico ambiental.

El comportamiento cosmovisivo holístico ambiental está dado por la conducta responsable del estudiante hacia sí mismo y su entorno inmediato, dirigido a transformar en la medida de lo posible las causas de los problemas ambientales, desde una perspectiva de sostenibilidad y adaptabilidad al impacto de los mismos, lo cual realiza a partir de la 
Estudios Pedagógicos XLVII Nº 1: 371-390, 2021

MODELO PEDAGÓGICO DE FORMACIÓN HOLÍSTICA AMBIENTAL EN LOS ESTUDIANTES DE CARRERAS PEDAGÓGICAS DEL ÁREA DE CIENCIAS NATURALES EN CUBA

aplicación de los saberes ambientales acerca de los diferentes objetos, fenómenos naturales y sociales con los que se relaciona, a la vez que es capaz de establecer comparaciones espacio-temporales, lo cual no está al margen de sus experiencias culturales ni de las características de su personalidad y el medio social.

El estudiante debe reflexionar acerca de la capacidad transformadora de la naturaleza humana, marcada por el saber hacer, el saber ser y el saber convivir, que se expresan en el entorno social y natural, cuyas características y normas éticas vigentes, también distinguen el comportamiento asumido. Especial atención se debe brindar al equilibrio entre los diversos factores que intervienen en el comportamiento, de modo que se logre un ambiente de satisfacción y de orden, a partir de la concertación de buenas prácticas, de ideas de intervención colaborativas que den solución oportuna a los conflictos en la diversidad de puntos de vista.

El comportamiento asumido en pro del cumplimiento de un objetivo debe producir efectos positivos, que faciliten su logro; de ahí la importancia de prevenir las consecuencias en el accionar cotidiano, pues cada persona evidencia a diario modos similares o diferentes de comportarse que constituyen la reproducción de las prácticas sociales que se manifiestan en la sociedad, lo cual demanda la sistemática comparación del presente y el pasado para la comprensión de la realidad actual, así como valorar las posibilidades futuras.

En este sentido, debe considerarse la diversidad de comportamientos que puede expresar el estudiante según el contexto, tener en cuenta sus intereses, aspiraciones y necesidades, lo cual proporcionará la reiteración de conductas correctas. Así las normas vigentes, permiten establecer dichas diferencias y precisar hasta qué punto el comportamiento individual o colectivo es apropiado según el contexto.

La interacción dialéctica entre las configuraciones consignadas como reflexión holística ambiental contextual, la capacidad proactiva holística ambiental y el desempeño pedagógico holístico ambiental, propicia el movimiento dialéctico, en la formación holística ambiental en los estudiantes de carreras pedagógicas, hacia la dimensión comportamental de la formación holística ambiental, la cual expresa un estado cualitativo superior de interpretación del proceso que se modela en su condición de totalidad y es muestra de las transformaciones existentes.

En la dimensión comportamental de la formación holística ambiental (Figura 3), el estudiante debe reflexionar acerca de lo que desea, debe y puede hacer, como base para realizar valoraciones acerca de lo correcto e incorrecto en la actividad humana, para evidenciar un comportamiento transformador en lo personal. Este comportamiento tiene también su expresión dialéctica en lo profesional y en lo individual, cuando se debe decidir entre lo que se quiere hacer y lo que se debe en correspondencia con un comportamiento cosmovisivo que evidencie su formación holística ambiental.

Debe potenciar en dichos estudiantes, un orden de vida individual y grupal, que sea justo, solidario, responsable, equilibrado y respetuoso de los límites en función del cuidado, protección y conservación de su entorno, así como sentir la necesidad de promover un clima de cooperación y construcción colectiva durante la realización de acciones ambientales en los diferentes contextos. También debe reconocer aquellos aspectos cognitivos y comportamentales que deben ser mejorados en cuanto a su propia formación ambiental, realizar valoraciones que los sitúen, no solo en mejores condiciones para responder a las exigencias de la profesión, sino que facilite una convivencia consigo mismo, con los demás y con el entorno en general, de forma más armónica y sostenible. 
Figura 3. Dimensión comportamental de la formación holística ambiental en los estudiantes de carreras pedagógicas del área de Ciencias Naturales.

Reflexión holística ambiental contextual

$\rightarrow$ Capacidad proactiva holística ambiental

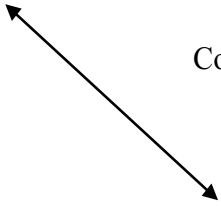

Comportamiento cosmovisivo holístico ambiental (Cualidad resultante)

\section{4}

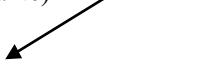

Dimensión comportamental de la formación holística ambiental

Desempeño pedagógico holístico ambiental

Fuente. Elaboración propia.

La estructura de relaciones entre las configuraciones y dimensiones del modelo pedagógico de formación holística ambiental en los estudiantes de carreras pedagógicas del área de Ciencias Naturales, en tanto se sustenta en el Enfoque Holístico Dialéctico, deviene en una configuración esencial, al revelar los movimientos, transformaciones de dicho proceso y así explicar, interpretar y predecir su comportamiento. Los nexos entre configuraciones y dimensiones se significan en un proceso armónico, los cuales constituyen relaciones dialécticas en el contexto, se integran a lo largo de la construcción teórica y como totalidad que se configura en la modelación que se describe en el actual texto, además, da cuenta de un proceso de naturaleza pedagógica, que se inserta en la formación del estudiante como se muestra en la figura 4.

Figura 4: Representación gráfica del modelo de formación holística ambiental en los estudiantes de carreras pedagógicas del área de Ciencias Naturales.

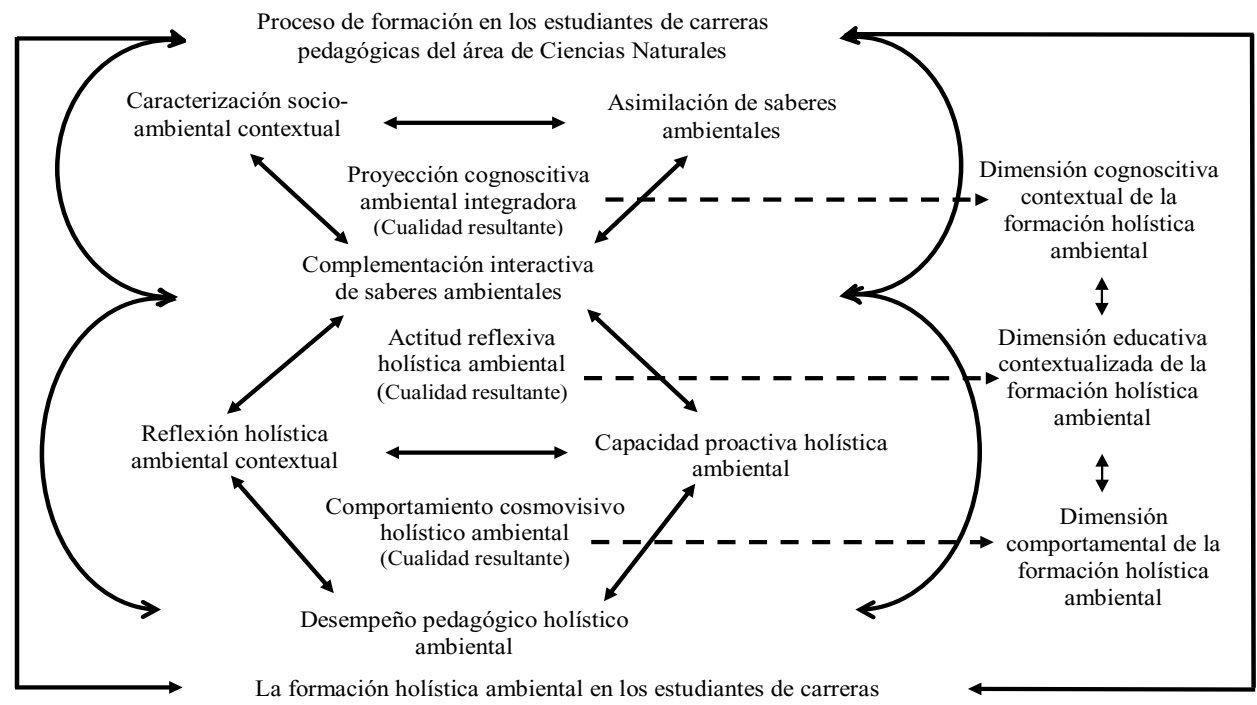

La formación holística ambiental en los estudiantes de carreras pedagógicas del área de Ciencias Naturales

Fuente. Elaboración propia. 


\section{DISCUSIÓN Y CONCLUSIONES}

El análisis de los referentes teóricos relacionados con la formación holística ambiental reveló la necesidad de la elaboración de un modelo pedagógico contentivo de las relaciones dialécticas de tesis, antítesis y síntesis entre las configuraciones que conforman cada dimensión, lo cual hace posible plantear las siguientes regularidades que determinan el movimiento para la formación holística ambiental en los estudiantes de carreras pedagógicas del área de Ciencias Naturales.

Al confrontar la caracterización socio-ambiental contextual con la asimilación de saberes ambientales, se propicia la complementación interactiva de saberes ambientales. A su vez, de esta última emergen la reflexión holística ambiental contextual y la capacidad proactiva holística ambiental, que se contrastan para sintetizarse en el desempeño pedagógico holístico ambiental. Se derivan de estas relaciones, como cualidades resultantes que se complementan en un nivel de ascenso progresivo: la proyección cognoscitiva ambiental integradora, la actitud reflexiva holística ambiental y el comportamiento cosmovisivo holístico ambiental. Tales configuraciones y sus rasgos, así como las cualidades, se articulan de forma armónica en las dimensiones: cognoscitiva contextual de la formación holística ambiental, educativa contextualizada de la formación holística ambiental y comportamental de la formación holística ambiental.

El proceso de formación holística ambiental a través del modelo pedagógico propuesto pudiera tener una concreción en la práctica educativa a partir de considerar las siguientes pautas metodológicas por los docentes: Indagación sistemática de las características del contexto socio- ambiental de los estudiantes, sobre la base del análisis y la reflexión que se derivan de la dinámica evolutiva en cada año académico. El estudio de las exigencias de los programas de asignaturas para contextualizar los contenidos relacionados con los procesos naturales y sociales según las vivencias de los estudiantes. La planificación por etapas de procedimientos metodológicos y acciones a implementar, de modo que se considere lo cognoscitivo, lo educativo y lo comportamental y que se contextualice de forma armónica en los procesos sustantivos de la universidad: académico, laboral, investigativo y extensionista.

También la instrumentación de las acciones concebidas en un ambiente favorable, caracterizado por el respeto a la diversidad, la cooperación, participación activa y voluntaria en los grupos de iguales. La socialización de ideas que expresen las alternativas teóricoprácticas o puntos de vistas, que han sido elaborados o meditados de forma individual o grupal. La comprobación valorativa de los avances progresivos, limitaciones que persisten, así como el análisis de la efectividad de las acciones implementadas.

\section{REFERENCIAS BIBLIOGRÁFICAS}

Busquets, T., Silva, M. \& Larrosa, P. (2016). Reflexiones sobre el aprendizaje de las Ciencias Naturales. Estudios Pedagógicos, Número Especial. 117-135.

CEPAL. (2015). Agenda 2030 y los Objetivos de Desarrollo Sostenible. Una oportunidad para América Latina y el Caribe. Organización de Naciones Unidas.

Cervantes, J. (2006). Estrategia educativa para la formación ambiental del ingeniero electricista. (Tesis doctoral). Santiago de Cuba: Universidad de Oriente. 
CITMA. (2017). Propuesta de directivas para el enfrentamiento al cambio climático. La Habana. . (2016). Estrategia Ambiental Nacional 2016-2020. La Habana.

Delgado, C. J. (2005). Bioética y medio ambiente. Cuba: Universidad de La Habana.

Díaz, R. (1998). La protección de la fauna silvestre mediante el proceso docente educativo en la Biología 2. (Tesis doctoral). Ciudad de La Habana: Instituto Superior Pedagógico Enrique José Varona.

Espino de Lara, R. (2013). Educación holística. Revista Iberoamericana de Educación.

Fernández, I. (2012). La formación ambiental del estudiante de la carrera psicología desde el proceso de extensión universitaria. (Tesis doctoral). Santiago de Cuba: Universidad de Oriente.

Figueroa, A. (1995). Formación ambiental. Revista Perspectivas Docentes. Universidad Autónoma de Tabasco. México.

Fuentes, H. C. (2011). La formación en la Educación Superior desde lo holístico, complejo y dialéctico de la construcción del conocimiento científico. Santiago de Cuba: Ediciones Universidad Oriente.

. (2004). La diversidad en el proceso de investigación científica. Reto cultural en la formación de investigadores. Santiago de Cuba: Universidad de Oriente.

Gallegos, R. y Verdugo, H. (2009). Educación holista. La pedagogía del amor universal. Fundación Internacional para la Educación Holista. Guadalajara.

García, R. (2017). La formación ambiental del docente del Centro Universitario Municipal San Luis (CUM) para la gestión medioambiental comunitaria. (Tesis doctoral). Santiago de Cuba: Universidad de Oriente.

Horruitiner, P. (2009). La universidad cubana: el modelo de formación. Cuba: Editorial Universitaria del Ministerio de Educación Superior, p. 9.

Labrada, Y. (2017). Concepción didáctica para el tratamiento de la Geografía local en la formación permanente de los profesores de Geografía. (Tesis doctoral). Santiago de Cuba: Universidad de Oriente.

Leff, E. (2000). Aprender a aprender la complejidad ambiental. Madrid: Ediciones Siglo XXI, s.a. de c.v .

Martínez, A. (2013). La formación ambiental inicial del maestro primario orientada al desarrollo agrosostenible en condiciones de montaña. (Tesis doctoral). Guantánamo: Tesis de Guantánamo.

Mc Pherson, M. (2004). La dimensión ambiental en la formación de docentes en Cuba. Una estrategia metodológica para su incorporación. (Tesis doctoral). La Habana: Instituto Central de Ciencias Pedagógicas.

Ministerio de Justicia (2019, 10 de abril). Constitución de la República de Cuba. La Habana: Gaceta Oficial No. 5 Extraordinaria, República de Cuba, p. 72.

Novo, M. (1998). La Educación Ambiental. Bases éticas conceptuales y metodológicas. España.

Parada, A. (2008). Estrategia educativa para la formación de actitudes ambientales en los estudiantes de Secundaria Básica. (Tesis doctoral). Santiago de Cuba: Instituto Superior Pedagógico Frank País.

Planos, E. (2014). Síntesis informativa sobre impactos del cambio climático y medidas de adaptación en Cuba. Instituto de Meteorología: Agencia de Medio Ambiente.

Rodríguez, Z. (2016). La formación de la actitud pedagógica ambiental en estudiantes de la carrera Licenciatura en educación Biología- Geografía. (Tesis doctoral). Santiago de Cuba: Universidad de Oriente.

Romero, E. V. y Parada, A. (2010). Estrategia curricular de educación ambiental para el desarrollo sostenible. Santiago de Cuba: Universidad de Ciencias Pedagógicas Frank País García.

Roque, M. (2003). Estrategia educativa para la formación de la cultura ambiental de los profesionales cubanos de nivel superior orientada al desarrollo sostenible. (Tesis Doctoral). La Habana. Instituto Superior Pedagógico Enrique José Varona.

Santos, I. (2002). Estrategia de formación continuada en educación ambiental para docentes. (Tesis doctoral). Santa Clara: Universidad Matha Abreu. 
Estudios Pedagógicos XLVII N 1: 371-390, 2021

MODELO PEDAGÓGICO DE FORMACIÓN HOLÍSTICA AMBIENTAL EN LOS ESTUDIANTES DE CARRERAS

PEDAGÓGICAS DEL ÁREA DE CIENCIAS NATURALES EN CUBA

Simões, A. S., Yanes, G. \& Alfonso, Y. (2018). Estrategia pedagógica de educación ambiental para el desarrollo sostenible en la formación del estudiante de carreras pedagógicas de Angola. Universidad y Sociedad, vol.10(5), 147-152. Recuperado de http://rus.ucf.edu.cu/index.php/rus

Sotolongo, P. L. (2002). Complejidad, sociedad y vida cotidiana. La Habana: I Seminario Bienal Internacional acerca de las Implicaciones Filosoficas, Epistemologicas y Metodologicas de la Teoria de la Complejidad.

Tamayo, C. (2015). La modelación científica: algunas consideraciones teórico metodológicas. Santiago de Cuba: Curso Pre- Evento Maestro y Sociedad.

Torres, E. (1996). Cómo lograr la educación ambiental de tus alumnos. La Habana: Editorial Pueblo y Educación.

Velasco, N. (2009). Bioética. Humanismo Científico Emergente. Fundación Cultural Javeriana de Artes Gráficas. Capítulo 4 Visión holística del ser. Bogotá, D.C.

Vigotsky, L. S. (1987). Historia del desarrollo de las funciones psíquicas superiores. La Habana: Editorial Científico Técnica. 